\title{
Erratum to: Excited States in P3HT and P3HT/PCBM Blends
}

\author{
K. Müller • M. Richter • S. Philip • M. Kunst • \\ D. Schmeißer
}

Published online: 7 March 2012

(C) Springer Science+Business Media, LLC 2012

Erratum to: BioNanoSci. (2012) 2:42-51

DOI 10.1007/s12668-011-0031-4

In the original article the $\pi$ and $\sigma$ symbols in Figs. 2, 3, and 4 did not appear correctly. Following are the corrected figures with their corresponding captions:

The online version of the original article can be found at http://dx.doi. org/10.1007/s12668-011-0031-4.

K. Müller $(\bowtie) \cdot$ M. Richter $\cdot$ S. Philip $\cdot$ D. Schmeißer

Brandenburgische Technische Universität Cottbus,

Angewandte Physik-Sensorik,

03046 Cottbus, Germany

e-mail: muellerk@tu-cottbus.de

M. Kunst

Helmholtz-Zentrum Berlin, Institut für Solare Brennstoffe (E-16),

Hahn-Meitner-Platz 1,

14109 Berlin, Germany 


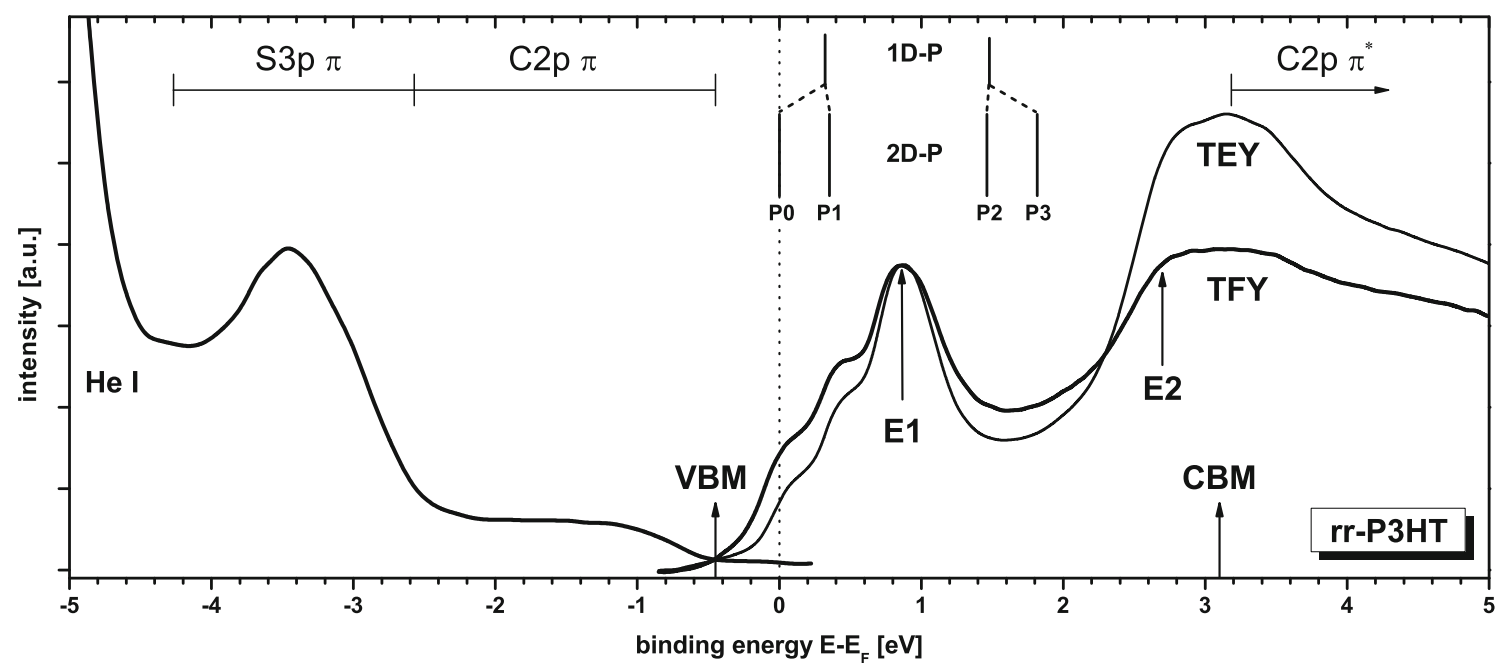

Fig. 2 Combined UPS-and C1s-XAS spectra of a rr-P3HT film, with respect to the Fermi level. The VBM and CBM are assigned as arrows; the features $E 1$ and $E 2$ are of excitonic; $\mathrm{P} 0,1,2$, and 3 are of polaronic nature, as described in text

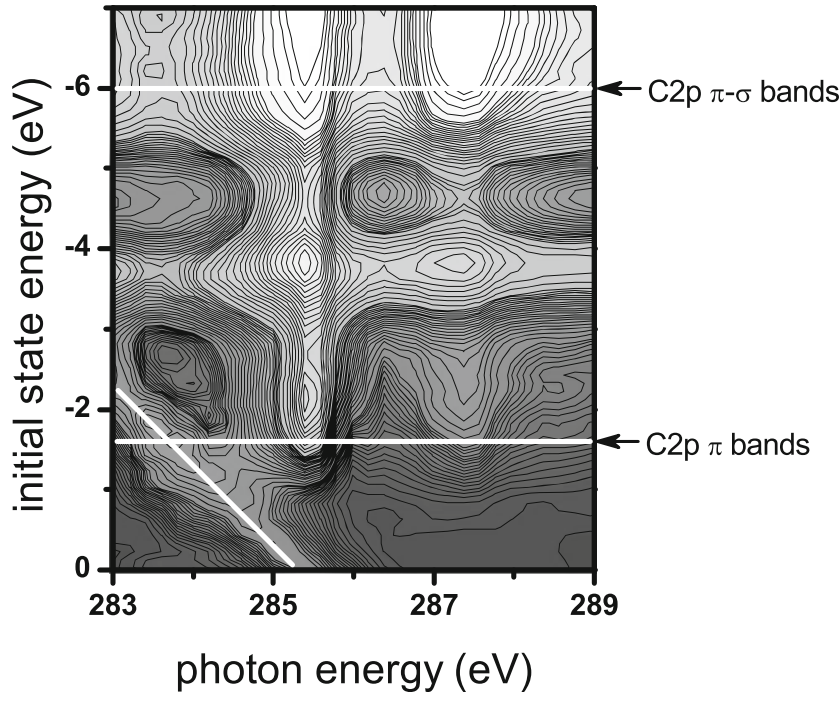

Fig. 3 2D resPES diagram of rr-P3HT on ITO. The photon energy is plotted as the $x$-axis, and the binding energy is plotted as $y$-axis. The intensity of the signal is given as a contour. Indicated are the two CIS cuts of Fig. 4 with an initial state energy of -1.6 and $-6.0 \mathrm{eV}$, corresponding to the $\mathrm{C} 2 \mathrm{p}-\pi$ and $\mathrm{C} 2 \mathrm{p}-\pi-\sigma$ bands, respectively. Also, the C1s XPS line excited by second-order light is marked, appearing in the lower initial state energy region

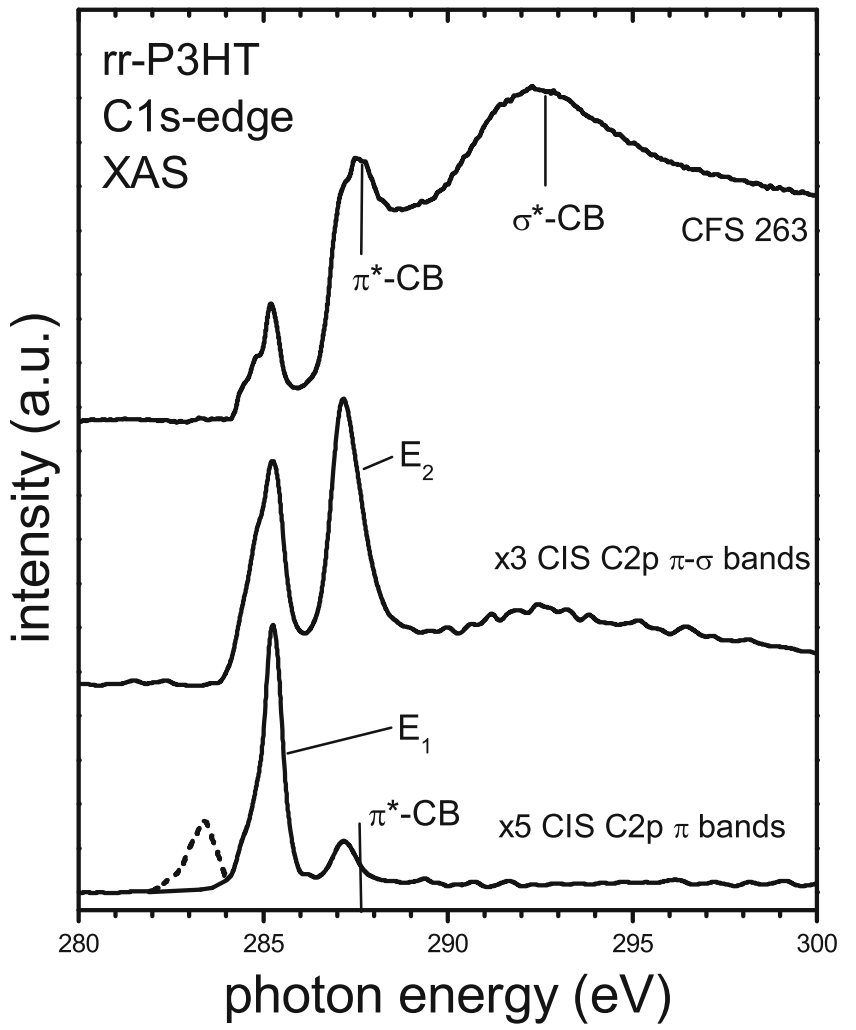

Fig. 4 XAS spectra in constant initial state mode (CIS), for different binding energies and in constant final state mode $(C F S)$, for different kinetic energies. The corresponding resPES plot is given in Fig. 3. The dashed part of the C2p- $\pi$ band is the C1s XPS line excited by secondorder light appearing in the lower initial state energy region of the $2 \mathrm{D}$ resPES spectra 